\title{
LXIX. On the luminosity of the human subject after death, with remarks and details of experiments made with a view of determining the nature of the fact
}

\author{
Mr. Daniel Cooper A.L.S. \& Mr. Robert Cooper
}

To cite this article: Mr. Daniel Cooper A.L.S. \& Mr. Robert Cooper (1838) LXIX. On the luminosity of the human subject after death, with remarks and details of experiments made with a view of determining the nature of the fact , Philosophical Magazine Series 3, 12:76, 420-426, DOI: $10.1080 / 14786443808649478$

To link to this article: http://dx.doi.org/10.1080/14786443808649478

曲 Published online: 01 Jun 2009.

Submit your article to this journal

Џ Article views: 2

Q View related articles $₫$ 
upon your notice, merely requesting their insertion at your earliest convenience.

I am, Gentlemen, yours, \&c.

New Bridge Street, April 13, 1838. Thomas Taylor, M.R.C.S.

LXIX. On the Luminosity of the Human Subject after Death, with Remarks and Details of Experiments made with a view of determining the nature of the fact. By Mr. DasieL Cooper, A.L.S., Curator to the Botanical Society of Lonilon, \&c., and Mr. Robert Cooper.**

$\mathrm{N}$ the 14th day of February, 1838, the body of William Tomkins, aged 88 , shoemaker by trade, was received at the Webb-street School of Anatomy and Medicine, Borough, having died of age and debility; and on the $3 \mathrm{rd}$ of March that of Robert Boreham, aged 45, was also received with the following history. It appeared that this individual, previous to his death, had been observed in the street in a state of extreme poverty, and was accordingly conveyed by a police officer on duty to the Station House, where, from extreme fatigue and exhaustion, the man died on the 26th of February. Being an unclaimed corpse, the parish authorities (according to the regulations of the Act of Parliament for supplying the Anatomical Schools with subjects for dissection) sent it to Webb-street. Previous to the reception of the latter, nearly the whole of the first subject had been dissected, and the only part which exhibited the luminous property was the left leg, which had been removed, according to custom, at the upper third of the thigh. Not having been informed of this phænomenon until it had been despatched for interment, we had not the opportunity of making experiments with regard to the cause.

We were, however, more fortunate with the latter subject, which presented the same appearance, but in a greater degree. Upon examination it was evident that the man had been a muscular and likewise a hard-working individual, if we might be allowed to judge from the appearance of the skin of the palms of the hands.

The phænomenon was first observed by Mr. J. Appleton, (the Curator to the above establishment, ) on Saturday the 3rd March, upon taking his accustomed round of an evening to every part of the building previous to retiring to rest. $\mathrm{He}$ was greatly surprised at perceiving the extremity before mentioned to be luminous; never having heard or witnessed in the whole course of his experience, commencing in 1812, a similar occurrence.

* Communicated by the Authors: see the Intelligence and Miscellaneous Articles in a future page. 
A few nights after the introduction of Boreham, Mr. Appleton observed this subject to be similarly affected, and the following morning communicated this fact to the Professors of the School. The circumstance having become generally known to the Pupils, several assembled the next evening for the purpose of observing this singular and novel phænomenon, when it was remarked by Mr. Appleton that the luminosity had considerably increased since its first appearance.

This novel fact we consider deserving the attention of physiologists, for in no work can we find recorded any notice of the phosphorescent or luminous appearance of the human subject. We are fully aware of its occurrence in many of the lower and even the higher classes of animals; but we are not aware of the present fact having been heretofore recorded.

Development of Light in the Lower Animals, and certain Substances.-Müller, the celebrated German physiologist, in a late edition of his work on the Elements of Physiology,(translated by W.Baly)makes some observations with respect to animals\&c., which possess the power of emitting a phosphorescent light. The following is but a brief abstract from the work. Müller commences with a description of the animals which produce the phosphorescence of the sea, and enumerates some of the Infusoria, Polipifera, Medusa, Annelides, Planaria, and Mollusca, mentioning occasionally some of the leading genera in each group. He then notices some of the leading phosphorescent crustaceans and insects, and mentions the opinion of Treviranus with respect to the light emitted from insects, viz. that the internal parts of generation are the source of light. He then further alludes to the opinion of Treviranus, viz. that light is derived from matter containing phosphorus, which is formed under the influence of light, but once formed is in some measure independent of light. He then brings forward the opinion of Carradori, Beccaria, and Monti as to the power of certain bodies of absorbing light during the day and emitting it during the evening, as is evidenced by several mineral substances, such as sulphate of barytes mixed with sulphuret of barium, oyster shells heated to redness with sulphur, \&c., and also by several organic substances when dried, such as seeds, flour, starch, acacia gum, quills, cheese, yolk of egg, muscle, tendon, isinglass, glue, and horn.

We areinformed, according to the observations of Mr. White, that he has repeatedly witnessed the luminous appearance of birds when they have been hanging for some time. Whilst we ourselves are aware of the fact, that many animals, such as dogs, cats, \&c., which have been killed, and left exposed to the atmosphere in ditches, \&c. have emitted a phosphorescent 
light; we have also been informed by Mr. Nazer that he has distinctly observed this luminous property in veal. In order further to verify the fact that it has been observed in several Mammalia, we have made diligent inquiries of several purveyors of meat in the metropolis, who have the opportunity of seeing meat in all its stages of decomposition, and we are informed, that they have at times perceived it on a dark night to be slightly luminous; but such a phænomenon is of rare occurrence. 'The common opinion with these individuals with respect to the cause, is, that the meat had been struck by lightning, it having been generally observed in the summer months.

On the parts of the Body most affected.-When the luminosity was discovered in Boreham, it was observed to occupy both the interior and exterior of the thorax, and gradually extended to other parts of the body, more especially the bones, tendons and fasciæ, and also to the muscles, but in a slighter degree. On Saturday, March 10th, we observed the cartilages and bones of the ribs extending from the fourth to the seventh on the right side, and on the back from the fifth to the ninth dorsal near their point of attachment to their vertebra: the light in the interior corresponded in situation to the light on the exterior of the thorax ; there was no phosphorescence observed on the viscera of the chest or abdomen. It likewise extended over the right and slightly over the left lumbar, sacral, and iliac regions as far down as the insertion of the tensor vaginæ femoris between the two laminæ of the fascia lata, from which fascia we were enabled to remove it with our fingers, and to them it gave a luminous appearance. On the evening of the following Monday we continued our researches. On entering the room we observed it greatly diminished in intensity; and upon examining the body we perceived the right knee (the integument of which had that day been removed) to be very luminous. Upon taking a scalpel and scraping the bone, we were surprised to find that the luminosity in no way diminished: although we could remove it by continual scrapings, it seemed to extend into the substance of the bones.

Power of increase. - In order to ascertain whether Boreham had been inoculated with the matter from Tomkins, we placed a portion of the luminous matter taken from the former on the chest of another subject,-situated on the opposite side and further end of the room, on Monday the 12th; and accordingly on Wednesday the 14th, as we anticipated, we discovered the trunk of the inoculated subject to be luminous to a very great extent. This occurrence clearly proves to our minds, that the matter from Tomkins had inoculated the body of Boreham. In order to ascertain whether the luminosity 
was situated in the moist or dry parts, we noted in the dark the situation of the luminosity; which upon examination in the light showed it to be the moistened parts.

Microscopic Observations. - With a view of elucidating this phænomenon we submitted a portion of the luminous matter scraped carefully from the subject to microscopic examination, in the first place, with the idea that from its exceedingly rapid augmentation it was due to an animal very low in the scale of organization. Our first examination led us to suppose, from the peculiar motion of some of the molecules in the fluid, that an animal of extreme minuteness was present : but upon further examination, with the assistance of Mr. Bowerbank's microscope and experience in these matters, we were convinced that no such animal as the Monas existed in the matter. It was not until we had an opportunity of witnessing the various but similar currents in a weak solution of gamboge, that we could reconcile ourselves to the appearance; for at times we observed small globules starting from one side to the other, and occasionally stemming the current for a considerable disstance. Until we had observed the similar motion in the gamboge, we did not feel perfectly satisfied that no living being there existed. In the course of the examination, Mr. Bowerbank observed a small threadlike body dart across the field of the microscope, which he immediately recognised as one of those bodies (Vibriones) which are so abuindantly seen upon macerating animal matter, such as a mouse, in water for a length of time. The power of the lens under which we observed the above was about 900 ; and speaking in general terms of the size of the molecules before mentioned, as viewed under the above power, they were as near as could be ascertained the 100,000th of an inch in size; so small indeed were they, that it was totally impossible to measure them with the finest micrometer as yet constructed. And according to the measurement of certain animals which among others produce the luminosity of the sea, as measured by Mr. Bowerbank at about 100 th of an inch, these molecules, for we will not give them any higher title, were at least 1000 times smaller. Although not exactly in connection with the present subject we have given the rough estimate of the molecules observed, as compared with the minute animals which are known to give the sea the beautiful phosphorescent appearance so frequently observed.

A portion of luminous matter having been placed under the microscope, the light evolved was sufficient to illuminate the field in patches. The luminosity appeared to be emitted from an oily matter.

Experiments with gases. - Having been led to suppose from 
the microscopic examinations that there were no traces of animals, we resolved to repeat the experiments of Macartney and Murray as regards the non-disappearance of the phosphorescent light emitted from animals in the different gases. For this purpose we prepared in well-stoppered phials the following gases, viz. oxygen, hydrogen, nitrogen, chlorine, carbonic acid, carbonic oxide, sulphuretted and phosphuretted hydrogen; and into a phial filled with each of these gases we introduced a portion of luminous muscle, tendon, or fascia for the space of 40 minutes, and the following were the results of the experiments :-

No effect observed in

Oxygen 7 remained lu-

Hydrogen minous for

Nitrogen $\int$ five days.

Carbonic oxide.

Phosphuretted Hydrogen.

Slight effect produced in

Carbonic acid.
Total extinction in Chlorine.

Sulphuretted hydrogen.

From the above experiments, we are compelled to disagree with the conclusions of Macartney and Murray as regards the non-disappearance of the phosphorescent light emitted from animals when immersed in the different gases. By perusing the above table, it will be observed that a total extinction of the light takes place when immersed in chlorine and sulphuretted hydrogen: this took place within the space of two minutes.

Appearance in vacuo.- On this point, we cannot coincide with the opinions of Macartney and Murray, having introduced an exceedingly luminous portion of flesh under the receiver of an air pump; and upon exhausting the vessel, the phosphorescence almost entirely disappeared, after having been in vacuo for the space of 15 minutes; but upon the readmission of the atmosphere, it immediately regained its former brilliancy, which is contrary to the opinions of the above experimentalists. Upon removing the portion of flesh from the phial containing carbonic acid, and placing it beneath the receiver of the air pump, and on first exhausting, it appeared to regain its luminosity in a slight degree; further exhaustion however diminished it. Oxygen having been admitted in the place of air, it soon regained its original brilliancy; this effect was in like manner produced by the admission of the atmosphere as before, and also by some of the different gases above-mentioned.

Effect in condensed air.-From the result of the foregoing experiment, viz. the diminution of the brilliancy upon withdrawing the atmosphere, we were led to suppose that a contrary effect would be produced upon condensing the air : to 
effect this, we procured a Cavendish bottle, in which a portion of luminous matter was placed. Upon using the condensing syringe, a visible increase of brilliancy occurred.

Luminous appearance under water.-Upon taking a portion of luminous flesh, and placing it in a glass of distilled water, it retained its luminosity for the space of from 10 to 15 minutes; and upon carefully removing the luminous matter from another portion of flesh with a knife, and agitating the water with the instrument, small globules of luminous matter were observed dispersed throughout the fluid, which remained for the space of 1 minute and a half.

Appearance in milk.-Upon treating the matter as in the preceding experiment, it gives to the fluid a very luminous appearance, which lasts from 15 to 20 minutes; the brilliancy dependent upon the quantity of matter introduced.

In Oil. - The luminous appearance remains in this medium for the space of three or four days. Upon rubbing the immersed flesh against the sides of the glass, it became more vivid.

In Alcohol.-Upon immersion in this fluid, it is extinguished in the space of two minutes. It does not impart to the alcohol the same appearance as is observed in the water or milk.

Heat.-Immediately extinguished, upon being placed in boiling water and heated air.

Cold.-U Upon placing a portion in a glass, and suspending the glass in a freezing mixture, no effect was observed after the lapse of 90 minutes.

Effects in the Diluted Mineral Acids.-Strength of solution 6 fluid drachms of acid to 2 fluid ounces of water.

Sulphuric Acid.-Extinguished almost immediately.

Nitric Acid.-Effect not so immediate as the preceding.

Muriatic Acid.-Not so immediate as the nitric.

Diluted Vegetable Acids. -Solution same proportions as above.

Acetic Acid.-Soon out after immersion.

Tartaric Acid.-Not so immediate as preceding.

Oxalic Acid.-Requires a longer period than tartaric. sion.

Diluted Alkalies.-Ammonia.-Extinguished on immer-

Potassa.-In this medium some time is required to extinguish the luminosity.

Muriate of Soda.-A strong solution of this substance extinguishes it almost immediately.

It would be difficult to state the true nature of the cause of this phænomenon. From our own observations, and the results 
of the above experiments, we are inclined to believe that it is the effect of a peculiar state of decomposition, totally independent of atmospheric causes, the luminosity residing (to the best of our belief,) in the oily matter, which we observed upon submitting it to microscopic examination : we hope however, that at some future period, we may have an opportunity of observing the same phænomenon, and continuing our researches.

82, Blackfriars Road, London.

\section{Proceedings of Learned Societies.}

ROYAL SOCIETY.

[Continued from p.368.]

Feb. 15, A Paper was in part read, entitled “ Experimental Re1838. A searches in Electricity," Twelfth Series, by Michael Faraday, Esq., D.C.L., F.R.S., \&c.

February 22.-The reading of a paper, entitled, " Experimental Researches in Electricity," T'welfth Series, by M. Faraday, Esq., D.C.L., F.R.S., was resumed.

March 1.-The reading of a paper, entitled " Experimental Researches in Electricity," Twelfth Series, by Michael Faraday, Esq., D.C.L., F.R.S., \&c., was resumed and concluded.

Experimental Researches in Electricity : Twelfth Series. By Michael Faraday, Esq., D.C.L., F.R.S., Fullerian Professor of Chemistry in the Royal Institution of Great Britain.

The object of the present series of researches is to examine how far the principal general facts in electricity are explicable on the theory adopted by the author, and detailed in his last memoir*, relative to the nature of inductive action. The operation of a body charged with electricity, of either the positive or negative kind, on other bodies in its vicinity, as long as it retains the whole of its charge, may be regarded as simple induction, in contradistinction to the effects which follow the destruction of this statical equilibrium, and imply a transit of the electrical forces from the charged body to those at a distance, and which comprehend the phenomena of the electric discharge. Having considered, in the preceding paper, the process by which the former condition is established, and which consists in the successive polarization of series of contiguous particles of the interposed insulating dielectric; the author here proceeds to trace the process, which, taking place consequently on simple induction, terminates in that sudden, and often violent interchange of electric forces constituting disruption, or the electric discharge. $\mathrm{He}$ investigates, by the application of his theory, the gradual steps of transition which may be traced between perfect insulation on the one hand, and perfect conduction on the other, derived from the

\footnotetext{
* See our present volume, p. 358.
} 